\title{
Altersarmut in der Europäischen Union
}

Richard Hauser

In der Europäischen Union der 27 entsteht nicht nur eine immer enger werdende Verflechtung der Wirtschaftssysteme, sondern auch der Sozialpolitiken. Seit Einführung der „Offenen Methode der Koordinierung“ werden die Ergebnisse der nationalen Systeme der sozialen Sicherung von der EU-Kommission verglichen und kommentiert. Die Bekämpfung von Armut im Alter, die sehr stark von der Ausgestaltung der Alterssicherungssysteme abhängt, ist ein Zielbereich. Der Beitrag zeigt die großen Unterschiede in der Höhe der Armutsquoten der Alten, und er arbeitet die Bedeutung von Mindestsicherungsregelungen für die Bekämpfung von Altersarmut heraus. Ohne deren Stärkung und Ausbau wird die Altersarmut in vielen Ländern ansteigen.

\section{Einführung}

Es scheint, als ob allmählich in der Europäischen Union (EU) ein Europäisches Sozialmodell Gestalt gewinnt (Eichenhofer 2007, S. 156). So werden beispielsweise in den Vertrag von Amsterdam auch die „Europäische Sozialcharta“ und die „Gemeinschaftscharta der sozialen Grundrechte der Arbeitnehmer" einbezogen und explizit sozialpolitische Ziele formuliert, die die Gemeinschaft berechtigen und verpflichten, die Sozialpolitik der Mitgliedstaaten zu unterstützen und zu ergänzen. Eines dieser Ziele ist die Bekämpfung der sozialen Ausgrenzung, die mit Armut einhergeht (ebd. S. $90 \mathrm{ff}$.).

Entsprechend dem im Vertrag von Maastricht beschlossenen Subsidiaritätsprinzip zählt der Bereich der Sozialen Sicherung aber zu jenen Feldern, die jedes Mitgliedsland allein regelt. Jedoch können einstimmig auch einheitliche EU-weite Regelungen beschlossen werden. Ein Instrument für die unilaterale und multilaterale Annäherung an ein konkreteres Europäisches Sozialmodell könnte die seit einigen Jahren eingeführte „Offene Methode der Koordinierung“ sein. Sie ermöglicht, die Sozialmodelle der Mitgliedsländer auf Basis eindeutig definierter Indikatoren und einheitlicher Statistiken zu vergleichen und im Zuge wechselseitigen Lernens, jedoch ohne rechtliche Verbindlichkeit, auf eine Annäherung der nationalen Ziele und Instrumente hinzuarbeiten (ebd. S. 110ff.; Hauser 2002). Die bisherigen Erfahrungen mit der Offenen Methode der Koordinierung im Feld der Alterssicherung zeigen, dass alle Mitgliedsländer ähnliche demografische, soziale und finanzielle Probleme haben. Eines davon ist die Altersarmut. Auf
Basis einer neuen, in allen EU-Ländern erhobenen Datenbasis ${ }^{1}$ kann das Ausmaß von Altersarmut in den 25 Ländern, die im Jahr 2004 schon Mitglied der EU waren, vergleichbar dargestellt werden.

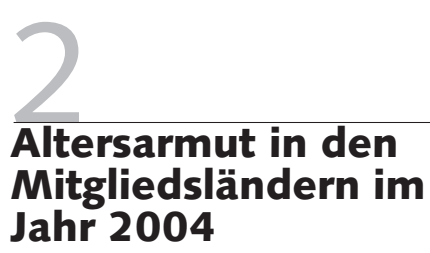

\subsection{ARMUTSVERGLEICHE - METHODISCHE PROBLEME}

In hochentwickelten Ländern mit marktwirtschaftlich orientierten Wirtschaftssystemen und ausgebauten, jedermann zugänglichen sozialen Sicherungssystemen können normale Bürger ein sozio-kulturelles Existenzminimum erreichen, wenn sie ein ausreichend hohes verfügbares Einkommen erzielen. Aber selbst ein Einkommen, das für Erwachsene im mittleren Lebensalter die Verwirklichung eines solchen Existenzminimums ermöglicht, kann für alte Menschen zu gering sein, wenn sie z. B. keine volle Beweglichkeit mehr besitzen oder mit größeren Gesundheitsproblemen zu kämpfen haben und hilfsbedürftig sind. Sollte sogar der Aufenthalt in einem Altersoder Pflegeheim erforderlich sein, so reicht ein derartiges minimales Einkommen erst recht nicht aus. Eigentlich müsste daher das Einkommen im Alter, das zur Deckung des sozio-kulturellen Existenzminimums erforderlich ist, um einen Zuschlag erhöht werden, der der individuellen Bedarfssituation entspricht.

Andererseits leben viele rüstige alte Menschen in ihrer eigenen Immobilie oder zusammen mit der nächsten Generation mietfrei in deren Wohnung oder Haus. Die ersparte Miete müsste eigentlich bei einem
Vergleich mit Mieterhaushalten als Einkommensbestandteil berücksichtigt werden. Bisher ist es aber nicht möglich, solche zusätzlichen Bedarfe und Einkommenskomponenten von alten Menschen statistisch in vergleichbarer Weise zu erfassen. Man ist deswegen darauf angewiesen, auf die verfügbaren Einkommen zurückzugreifen und diese zu vergleichen; zusätzliche individuelle Bedarfe wie auch Einsparmöglichkeiten (wie z. B. das mietfreie Wohnen) bleiben dabei außer Acht.

Ein weiteres Problem bei einem Vergleich der Armutsquoten von alten Menschen in den EU-Mitgliedsländern ist, dass Personen, die in Einrichtungen leben - insbesondere jene, die in Alters- oder Pflegeheimen untergebracht sind - überhaupt nicht berücksichtigt werden. Die Aussagekraft der folgenden Einkommensvergleiche ist daher eingeschränkt.

Die Offene Methode der Koordinierung stützt sich zur Erfassung von Armut auf einheitlich definierte Indikatoren, die

\footnotetext{
1 Die einheitliche Datenbasis, die nach Richtlinien von Eurostat in allen Mitgliedsländern jährlich erstellt wird, heißt "European Statistics on Income and Living Conditions (EU-SILC) “. Sie wird künftig auch der Wissenschaft für weitere Analysen zur Verfügung gestellt. Die Abbildungen 1-6 beruhen auf Tabellen, die die Europäische Kommission (Commission of the European Communities 2007) veröffentlicht hat.
}

Richard Hauser, Prof. Dr., emeritiert, lehrte Volkswirtschaftslehre, insbesondere Sozialund Verteilungspolitik, an der Johann Wolfgang Goethe-Universität, Frankfurt am Main; Arbeitsschwerpunkte: Systeme der sozialen Sicherung im internationalen Vergleich, Einkommens- und Vermögensverteilung, Armutsforschung. e-mail: r.hauser@em.uni-frankfurt.de 
auf dem verfügbaren Einkommen basieren (Atkinson et al. 2002; Marlier et al. 2007). Die Grenze der Einkommensarmut wird dabei nicht etwa nach einem für alle EULänder gleich hohen Eurobetrag bemessen, sondern sie wird relativ zum mittleren Einkommen (Median) in jedem Mitgliedsland definiert. Der Vergleich beruht also auf landesspezifischen relativen Einkommensarmutsgrenzen, die mit zwei unterschiedlichen Argumenten begründet werden: Erstens wird unterstellt, dass sich jedes Gesellschaftsmitglied mit den anderen Mitgliedern der gleichen Gesellschaft vergleicht und dass auch die Beurteilung einer Person durch andere von einem derartigen relativen Vergleich geprägt ist. Soziale Exklusion hängt also unter anderem davon $\mathrm{ab}, \mathrm{ob}$ man wenigstens einen Lebensstandard erreicht, der in dem betreffenden Land als Minimum bzw. landesspezifisches sozio-kulturelles Existenzminimum angemessen erscheint. ${ }^{2}$ Das zweite Argument betrifft die Erfolgswirksamkeit sozialpolitischer Maßnahmen zur Bekämpfung von Armut. Da die im Durchschnitt erreichten ökonomischen Niveaus der 27 Mitgliedsländer weit auseinanderklaffen, würden bei einer absolut einheitlichen Armutsgrenze die daran orientierten Mindestsicherungsleistungen in einem ärmeren Mitgliedsland über den mittleren Löhnen liegen und damit den Anreiz zur Sicherung des Lebensunterhalts durch eigene Arbeitsleistung und zur Verbesserung des Ausbildungsstandes stark mindern; demgegenüber würden sie es in einem reicheren Mitgliedsland nicht erlauben, das dortige sozio-kulturelle Existenzminimum $\mathrm{zu}$ sichern.

Abbildung 1 zeigt die Unterschiede im wirtschaftlichen Niveau zwischen den Mitgliedsländern. Im Jahr 2005 lag das Bruttoinlandsprodukt pro Kopf - und umgerechnet zu Kaufkraftparitäten - von acht Ländern nahe bei oder über $120 \%$ des EUDurchschnitts. Demgegenüber gab es sechs Länder, deren Bruttoinlandsprodukt pro Kopf weniger als $60 \%$ des EU-Durchschnitts betrug. Die übrigen Länder lagen in der Zwischenzone.

Zur Bestimmung der EU-Armutsrisikogrenze und des Medians der Einkommensverteilung wird das sogenannte Nettoäquivalenzeinkommen der Personen verwendet. Es ergibt sich in mehreren Rechenschritten aus dem Nettoeinkommen jedes Haushalts, das sich wiederum aus der Summe aller Markteinkommen zuzüglich aller
Abb. 1: Bruttoinlandsprodukt pro Kopf in Kaufkraftparitäten im Verhältnis zum EU 25-Durchschnitt im Jahr 2006 - in \% -

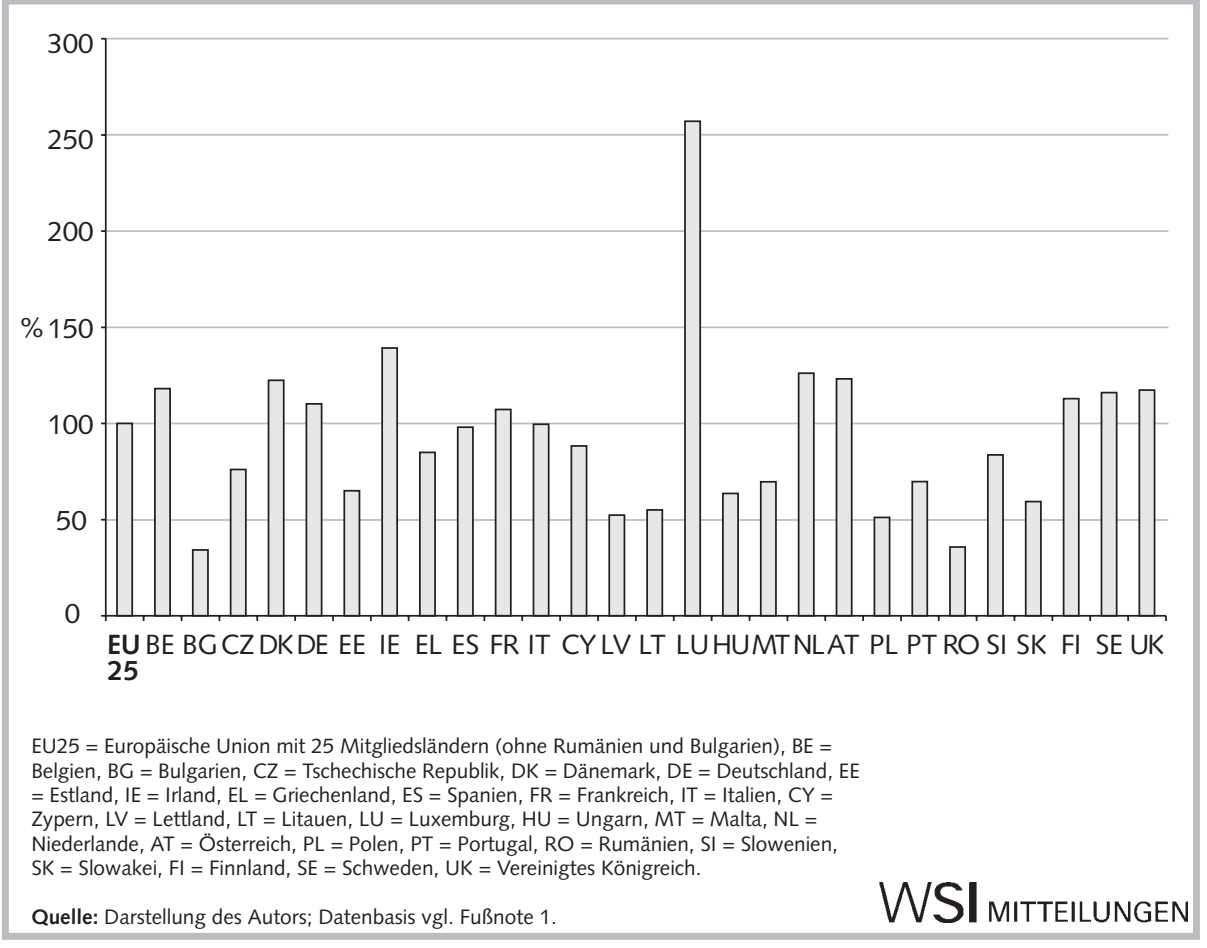

Sozialleistungen und abzüglich der Sozialversicherungsbeiträge sowie der Lohn- und Einkommensteuer der Haushaltsmitglieder zusammensetzt. Dieses Nettoeinkommen wird dann durch die Summe der Äquivalenzgewichte aller Haushaltsmitglieder dividiert und jedem Haushaltsmitglied zugeordnet. Es handelt sich also um ein gewichtetes Pro-Kopf-Nettoeinkommen. Das Gewichtungsschema wird als Äquivalenzskala bezeichnet. Die von der EU verwendete Skala verleiht dem ersten Erwachsenen im Haushalt ein Gewicht von 1,0 , weiteren Personen über 13 Jahre Gewichte von 0,5 und jüngeren Kindern Gewichte von $0,3 .{ }^{3}$ Begründet wird ein derartiges Gewichtungsschema zum einen damit, dass durch die Wirtschaftsgemeinschaft eines gemeinsamen Haushaltes Einsparungen erzielt werden können; zum anderen, dass jüngere Kinder einen geringeren (Verbrauchs)Bedarf als Erwachsene hätten.

Werden alle Personen nach der Höhe ihres Nettoäquivalenzeinkommens in aufsteigender Reihe angeordnet, dann ist der Medianwert jenes Äquivalenzeinkommen, das genau in der Mitte liegt und daher die Bevölkerung in zwei gleich große Hälften teilt. Die von der EU festgelegte Armutsrisikogrenze bemisst sich dann als $60 \%$ des Medians; Personen, deren Nettoäquivalenzeinkommen unterhalb dieser Grenze liegt, werden als arm angesehen. Auf diese Weise wird auch Altersarmut in allen EU-Ländern als relative Einkommensarmut gemessen und vergleichbar gemacht.

2 Der Rat der EU hat im Jahr 1984 eine Definition von Armut beschlossen, nach der Personen, Familien und Gruppen als arm gelten, „die über so geringe (materielle, kulturelle und soziale) Mittel verfügen, dass sie von der Lebensweise ausgeschlossen sind, die in dem Mitgliedsstaat, in dem sie leben, als Minimum annehmbar ist" "Bundesministerium für Arbeit und Sozialordnung 2001, S. 7).

3 Diese von der EU verwendete Äquivalenzskala wird als modifizierte OECD-Skala bezeichnet. Die ursprüngliche OECD-Skala, die bisher in deutschen Armutsuntersuchungen verwendet wurde, weist Gewichte von 1,0, 0,7 und 0,5 auf. Sie entspricht den in vielen nach Haushaltsgröße abgestuften sozialpolitischen Regelungen implizit enthaltenen Äquivalenzskalen besser als die modifizierte OECD-Skala. Beispielsweise impliziert eine Hinterbliebenenrente von $60 \%$ der Rente des Versicherten als Alleinverdiener ein Äquivalenzgewicht von 0,66 . Wenn die Hinterbliebenenrente lediglich $55 \%$ beträgt, beläuft sich das implizite Äquivalenzgewicht sogar auf 0,8 (Hauser 1996). Letztlich führt die modifizierte OECD-Skala zu einer Unterschätzung des Bedarfs von Kindern und damit zu einer zu günstigen Darstellung der Lage von Alleinerziehenden und von Familien mit mehreren Kindern. Umgekehrt wird die Lage von Alleinstehenden und Paaren ohne Kinder zu ungünstig dargestellt. Dies ist auch bei den hier referierten Ergebnissen über die Altersarmut zu berücksichtigen. 


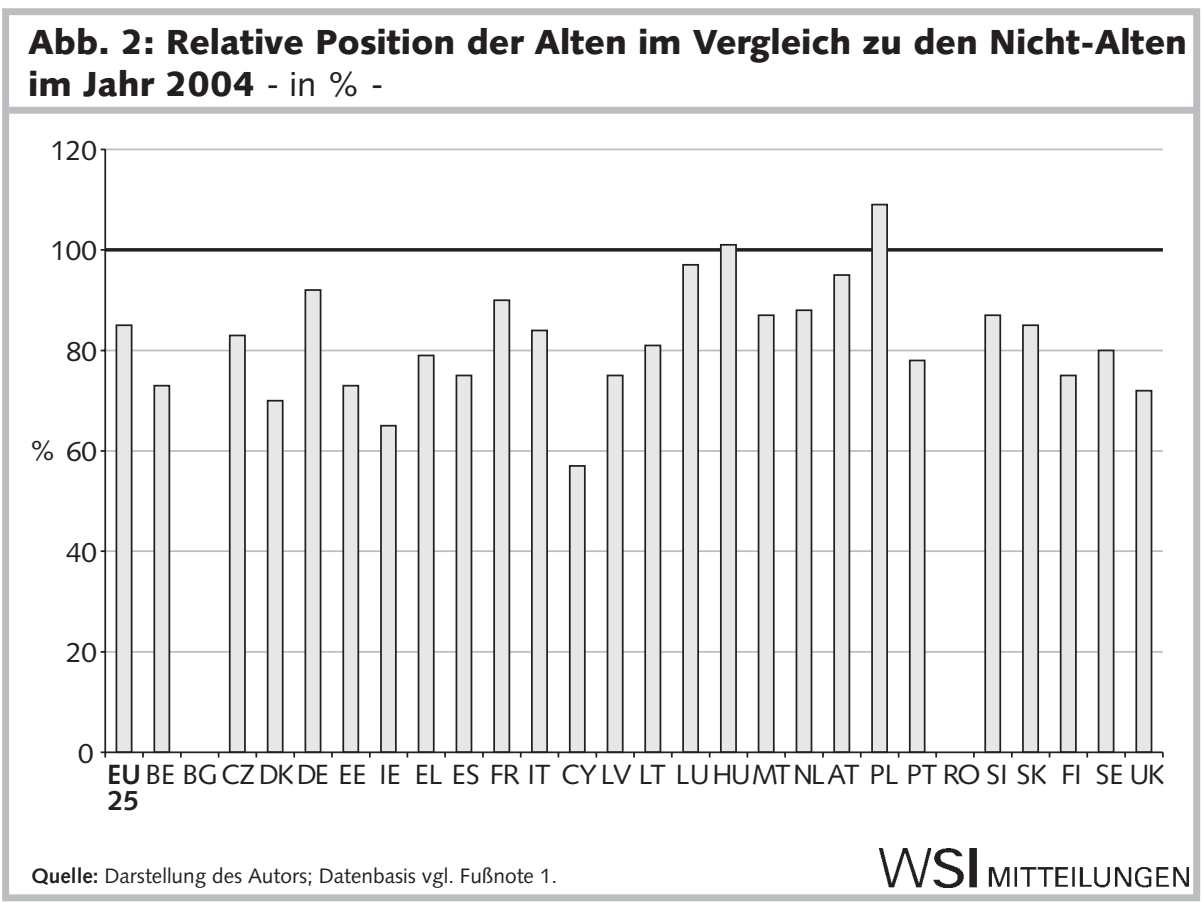

\subsection{ZUR RELATIVEN EINKOMMENS- POSITION DER ALTEN MENSCHEN}

Einen ersten Eindruck von der Einkommenssituation der alten Menschen in den Mitgliedsländern der EU erhält man, wenn man ihr durchschnittliches Nettoäquivalenzeinkommen zum Durchschnitt der Bevölkerung im Alter von 0 bis 64 Jahren - also der Nicht-Alten - ins Verhältnis setzt. $A b$ bildung 2 zeigt die Unterschiede zwischen den Mitgliedsländern. Nur in zwei Ländern (Polen und Ungarn) erreichen die Alten im

Abb. 3: Armutsquoten der Gesamtbevölkerung und der Alten (65+) im Jahr 2004 - in \% -

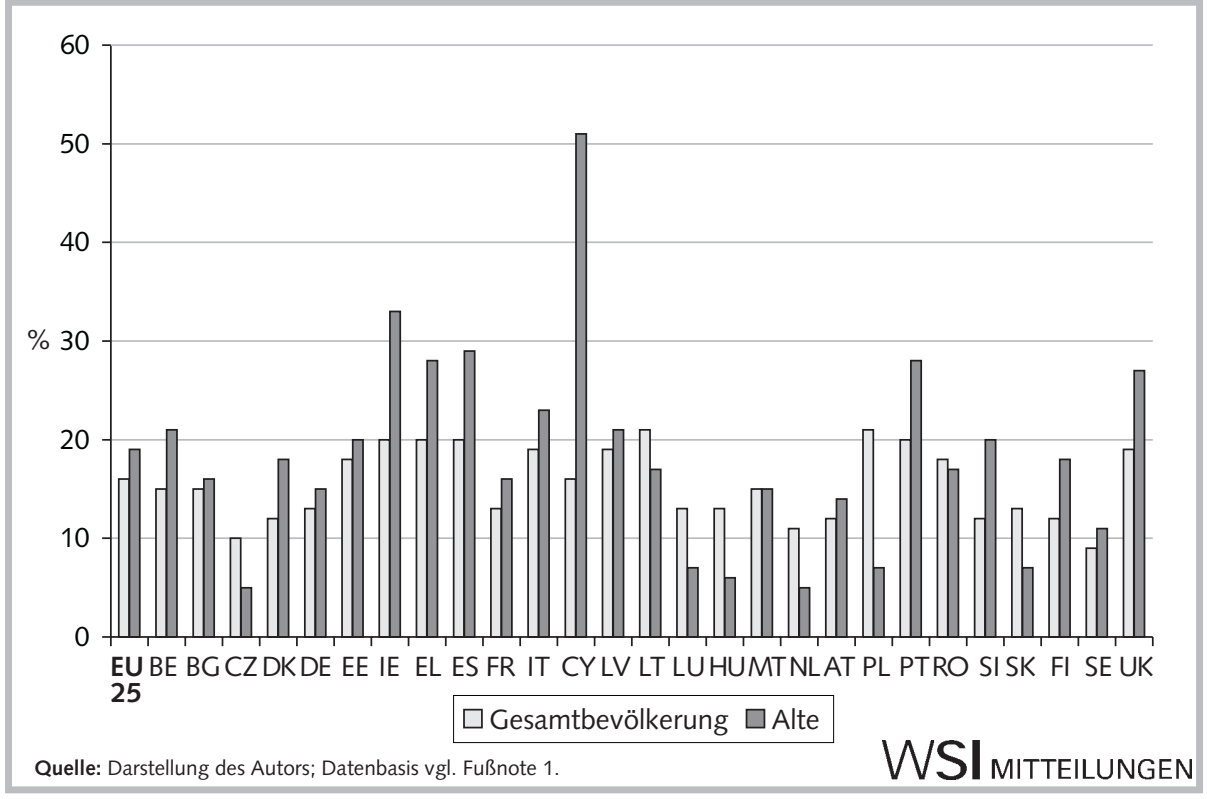

\subsection{ERGEBNISSE DER ARMUTS-} MESSUNG

\section{ARMUTSQUOTEN}

In Abbildung 3 wird die Armutsquote der gesamten Bevölkerung mit der Armutsquote unter den alten Menschen verglichen, die 65 Jahre und älter sind. Danach reicht bei einer durchschnittlichen Armutsquote in der EU von $16 \%$ für die Gesamtbevölkerung die Spannweite der Armut von $9 \%$ bis $21 \%$. Besonders niedrige Armutsquoten bis $13 \%$ weisen zwölf Länder auf: Tschechien, Dänemark, Deutschland, Frankreich, Luxemburg, Ungarn, die Niederlande, Österreich, Slowenien, die Slowakei, Finnland und Schweden - also sowohl alte als auch neue EU-Mitgliedsländer. Aber nur in Tschechien, Luxemburg, Ungarn, in den Niederlanden und in der Slowakei sind die Alterssicherungssysteme so effizient ausgebaut, dass die Armutsquoten der Alten noch unter den Quoten der Gesamtbevölkerung liegen. In den übrigen Ländern sind die Armutsquoten der Alten höher.

Demgegenüber finden sich die höchsten Armutsquoten der Gesamtbevölkerung mit $20 \%$ und mehr in Irland, Griechenland, Estland, Litauen, Polen und Portugal. Nur in Litauen und Polen ist die Armutsquote der Alten niedriger als die der Gesamtbevölkerung. In den vier übrigen Ländern dieser Gruppe liegen die Armutsquoten der Alten um 8 bis 12 Prozentpunkte darüber. Hier scheinen die Alterssicherungssysteme Altersarmut also nicht einmal auf das ohnehin sehr hohe Ausmaß der Armut innerhalb der Gesamtbevölkerung vermindern zu können. Bei einer dritten Ländergruppe mit mittlerer Höhe der Armutsquoten der Gesamtbevölkerung zeigt sich, dass die Quote der Alten fast immer höher ist. Besonders groß ist der Unterschied in Zypern mit etwa 35 Prozentpunkten.

Insgesamt gesehen ergibt sich also ein recht gemischtes Bild, das kein einfaches Muster zeigt. An dieser Stelle müssen aber zwei Einschränkungen betont werden: Erstens ist die erste Welle von EU-SILC trotz aller Anstrengungen von Eurostat noch nicht in jeder Hinsicht ausgereift. Manche Ungenauigkeiten könnten sich noch auf die Ergebnisse auswirken. Zweitens verwendet die EU wie gesagt eine Äquivalenzskala, die den weiteren Personen im Haushalt recht geringe Gewichte verleiht. Oder 
anders ausgedrückt: Sie unterstellt recht hohe Einsparungen beim gemeinsamen Wirtschaften und einen ziemlich geringen Bedarf von Kindern. Würden die Gewichte für weitere Personen im Haushalt erhöht - wie es den institutionellen Regelungen in den meisten EU-Ländern besser entspräche -, dann würden größere Haushalte als schlechter gestellt erscheinen und kleinere Haushalte, insbesondere die der Alten, würden besser abschneiden. Dies bedeutet, dass in Abbildung 3 die Armutsquoten der Alten überschätzt und die Armutsquoten der Nicht-Alten unterschätzt sein könnten. Es bedürfte jedoch neuer Berechnungen, um das genaue Ausmaß dieser Verschiebung zu ermitteln.

\section{GENDERDIFFERENZEN}

Im Sinne von Gender Mainstreaming werden von Eurostat die Armutsquoten jeweils auch für Männer und Frauen separat erfasst. Dabei muss man aber bedenken, dass die in einem Haushalt zusammenlebenden Männer und Frauen wegen der Ermittlung des Nettoäquivalenzeinkommens als gewichtetes Pro-Kopf-Einkommen immer die gleiche Position aufweisen, also entweder beide arm oder beide nicht arm sind. Die in Abbildung 4 sichtbar werdenden Unterschiede zwischen Männern und Frauen gehen also ausschließlich auf die alleinstehenden Alten zurück, d.h. auf Witwen und Witwer, Geschiedene, Getrenntlebende und Personen, die zeitlebens ledig waren.

Man sieht, dass im EU25-Durchschnitt und in den meisten Ländern Frauen eine höhere Armutsquote aufweisen als Männer. Mehr als fünf Prozentpunkte Unterschied bestehen in 15 von 27 Ländern, und zwar in Bulgarien, Deutschland, Estland, Irland, Spanien, Italien, Zypern, Lettland, Litauen, Österreich, Rumänien, Slowenien, Slowakei, Finnland und Schweden. Die Armutsquoten der Frauen sind in einigen Ländern sogar mehr als doppelt so hoch wie die der Männer. Dies gilt für Bulgarien, Tschechien, Estland, Lettland, Litauen, Slowenien, Slowakei und Finnland. Nur in Malta und Portugal sind die geschlechtsspezifischen Armutsquoten gleich groß, während Luxemburg das einzige Land ist, in dem die Armutsquote der alten Männer höher liegt als die der Frauen. Diese Unterschiede können hier nur aufgezeigt werden. Für eine intensivere Analyse der länderspezifischen Ursachen müsste

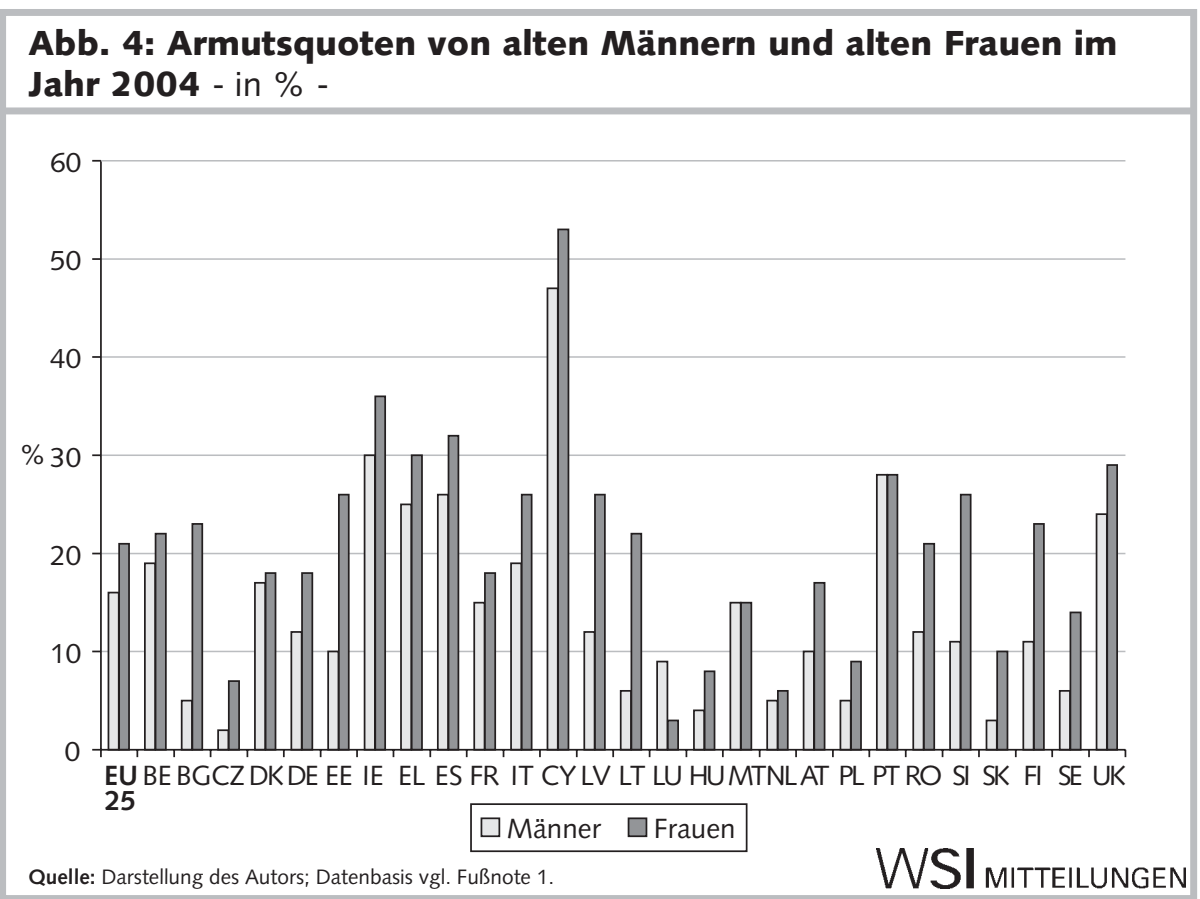

Abb. 5: Relative Armutslücke (bezogen auf Mediane) bei alten Männern und alten Frauen im Jahr 2004 - in \% -

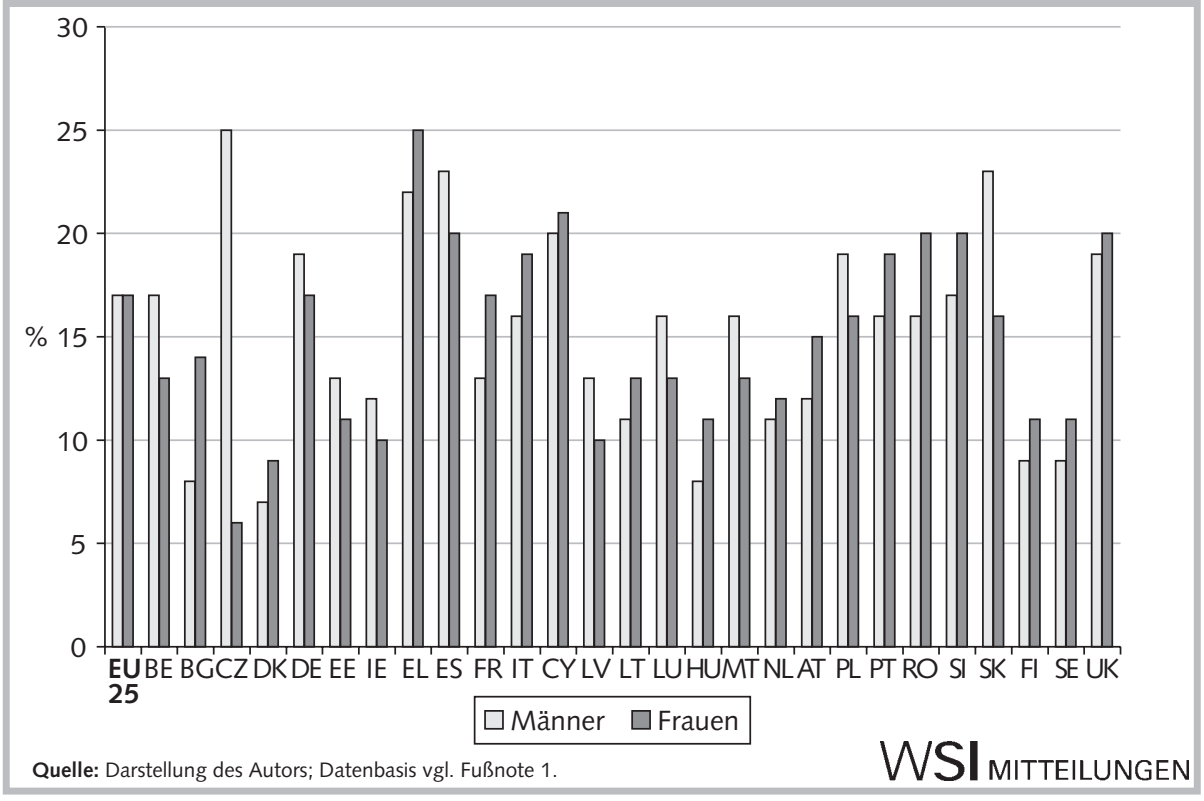

man die Rentenregelungen für Hinterbliebene, die Rechtsfolgen von Scheidungen und die Unterschiede zwischen den Frauen- und Männerlöhnen untersuchen. Auch die in den einzelnen Alterssicherungssystemen enthaltenen Mindestrenten und andere bedarfsorientierte Mindestsicherungsleistungen könnten direkt oder indirekt geschlechtsspezifisch ausgerichtet sein.

Die Höhe der Armutsquote ist aber nicht allein entscheidend für das Bild der Altersarmut. Es kommt auch darauf an, wie weit genau unterhalb der Armutsgrenze die Armen leben müssen. Zur genaueren Bestimmung dieses Abstandes vergleicht man das Medianeinkommen der armen Männer und Frauen mit der Armutsgrenze. Je größer dieser Abstand ist - die sogenannte Armutslücke-, desto schlechter geht es den betreffenden armen Alten. Drückt man diesen Abstand als Prozentsatz der Armutsgrenze aus, dann erhält man die in Abbildung 5 ausgewiesenen Ergebnisse.

Demnach gibt es kein klares Armutslücken-Muster zugunsten von Männern 


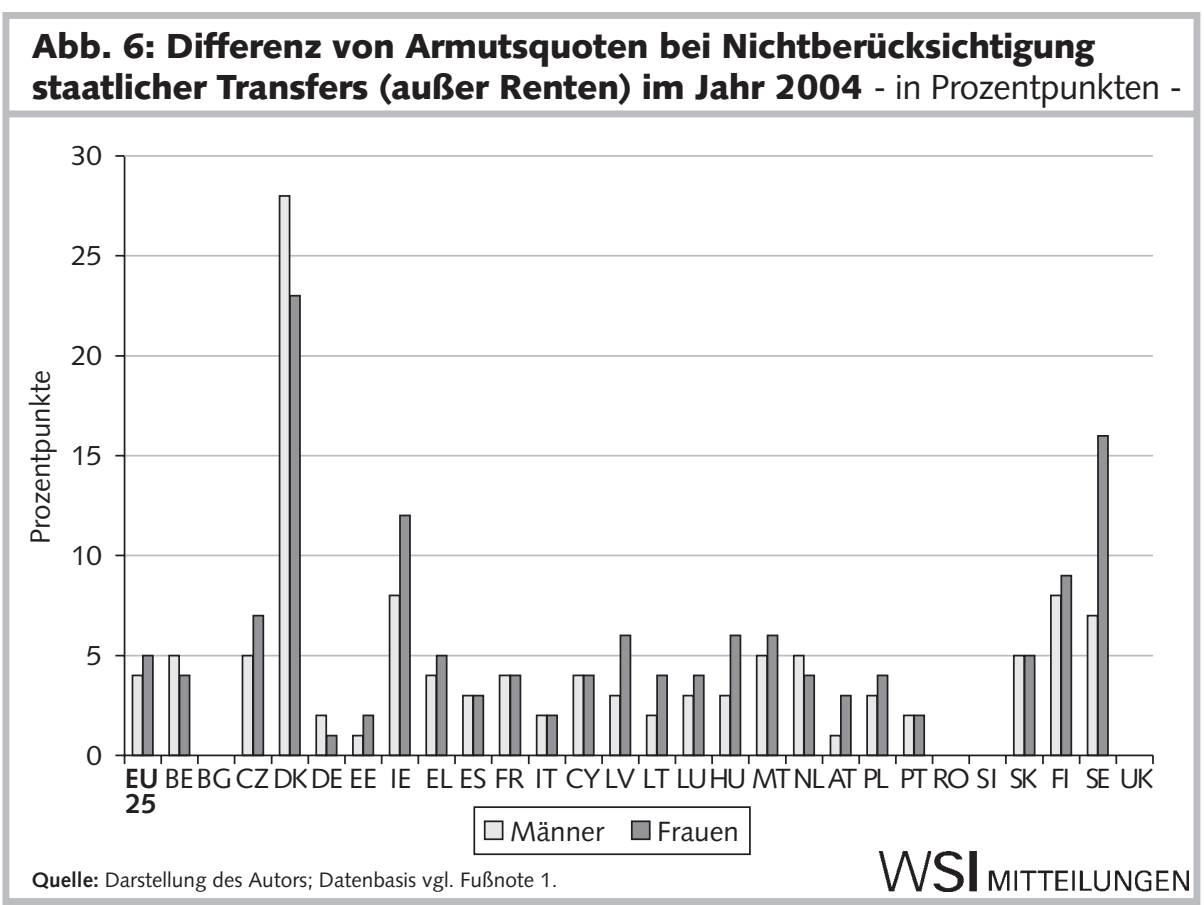

oder Frauen. In manchen Ländern liegen die Frauen weit unterhalb der Armutsgrenze, in anderen die Männer. Insgesamt gesehen ist die Armutslücke im EU25-Durchschnitt mit $17 \%$ für Männer und Frauen gleich groß. Das bedeutet, dass die armen Alten mit einem Einkommen auskommen müssen, das im Mittel um knapp ein Fünftel unter der von der EU festgelegten Armutsrisikogrenze liegt.

\section{ALTERSEINKOMMEN}

Die Einkommen der alten Menschen stammen aus verschiedenen Quellen. Sie umfassen Leistungen der Pflichtalterssicherungssysteme, Zahlungen von obligatorischen oder freiwilligen Zusatzsicherungen, Betriebsrenten, private Vermögenseinkommen sowie Leistungen von Mindestsicherungssystemen - wie der Sozialhilfe - und Mietbeihilfen, die bei zu niedrigem eigenem Einkommen gewährt werden. Man gewinnt daher einen weiteren Einblick in die Unterschiede der institutionellen Struktur der Mitgliedsländer, wenn man fiktive Armutsquoten errechnet, die sich ergeben würden, wenn nur Renten gewährt würden. Abbildung 6 zeigt, um wieviel die Armutsquoten der Alten ansteigen würden, wenn außer den Renten alle anderen staatlichen Transfers wegfielen. Zwar hängen diese Ergebnisse auch etwas davon ab, wie man die einzelnen Sozialleistungen klassifiziert, doch man kann feststellen, dass die Armutsquoten im
Durchschnitt bei den Männern um etwa vier und bei den Frauen um etwa fünf Prozentpunkte ansteigen würden. Das heißt aber, die Alterssicherungssysteme allein können in keinem Land Armut vermeiden, und zwar bei den Frauen noch weniger als bei den Männern. Es bedarf zusätzlicher Sozialleistungen.

Im Prinzip könnten die zusätzlichen, in der Regel bedarfsabhängigen und einkommensüberprüften Leistungen so hoch sein, dass Armut unter alten Menschen - gemessen an der EU-Armutsrisikogrenze - völlig vermieden wird. Dies ist aber offensichtlich nicht der Fall. Das Ausmaß, in dem die von den Alterssicherungssystemen sozusagen „übriggelassene“ Armut durch zusätzliche Sozialleistungen reduziert wird, ist aber von Land zu Land sehr unterschiedlich. Fast in allen Ländern werden die Armutsquoten von Frauen durch die sonstigen Sozialleistungen stärker reduziert als jene der Männer. Die größte Wirkung haben die sonstigen Sozialleistungen in Dänemark, Irland, Finnland und Schweden, die geringsten Effekte zeigen sich in Deutschland, Estland, Italien und Portugal. Mindestsicherungsleistungen außerhalb des Rentensystems haben auch im Vereinigten Königreich eine große Bedeutung für die Armutsbekämpfung, aber hierfür sind offenbar noch keine vergleichbaren Daten verfügbar.

\section{Mindestsicherungs- leistungen: Wichtige Elemente in Alterssicherungssystemen}

Die Ursachen von Altersarmut sind sehr vielfältig. Wenn die dominierenden Elemente eines Alterssicherungssystems eine stark am Äquivalenzprinzip orientierte Sozialversicherung oder eine obligatorische private Zusatzversicherung sind, deren spätere Renten auf Beitragszahlungen und Versicherungszeiten beruhen, dann kommen vor allem folgende Tatbestände vor dem Renteneintritt als Armutsrisiken infrage:

- Fehlende Universalität der Alterssicherungssysteme, d. h. der Ausschluss bestimmter Personengruppen vom Pflichtalterssicherungssystem;

- niedrige Erwerbseinkommen;

- längere Perioden der Arbeitslosigkeit;

- vorzeitige Erwerbsunfähigkeit infolge von Privatunfällen und Berufsunfällen oder Erwerbsunfähigkeit von Geburt an;

- längere Krankheiten;

- Scheidungen;

- freiwilliger Verzicht auf Erwerbsarbeit und

- Zuwanderung im mittleren Alter, ohne dass bereits ausreichende Alterssicherungsansprüche erworben und mitgebracht wurden.

Wenn das Alterssicherungssystem jedoch eine universelle Basisrente gewährt, die nur von der Aufenthaltsdauer als Steuerbürger abhängt, dann sind - mit Ausnahme der Zuwanderung - alle diese Tatbestände irrelevant. Es hängt dann lediglich von der Höhe dieser Basisrente im Vergleich zur Armutsgrenze ab, ob Altersarmut eintritt. Derartige Basisrenten gewähren beispielsweise die Niederlande und Dänemark, aber offenbar reicht deren Höhe allein nicht aus, um Altersarmut zu vermeiden. Es müssen Zusatzleistungen hinzukommen.

In den meisten EU-Ländern dienen universelle, d. h. prinzipiell die gesamte Bevölkerung einbeziehende Mindestsicherungsregelungen als unterstes Auffangnetz zur Bekämpfung von Armut oder speziell von Altersarmut. Die Ausgestaltungsformen und die Leistungsniveaus sind innerhalb der EU sehr unterschiedlich. Man kann drei Typen unterscheiden: 
(1) die bereits erwähnten Basisrenten, die nicht vom Lebenslauf vor Renteneintritt, sondern nur von der Dauer des Aufenthaltsstatus als Inländer abhängen und die in der Regel in die Rentensysteme integriert sind;

(2) Regelungen, die eine Einkommensund/oder Vermögensüberprüfung voraussetzen und nur aufstockende Leistungen gewähren; das anzurechnende Einkommen kann auf das eigene Renteneinkommen oder das Renteneinkommen des Ehepaars beschränkt oder umfassend definiert sein. In Deutschland dient die Bedarfsorientierte Mindestsicherung im Alter und bei Erwerbsminderung, die im SGB XII geregelt ist, der Armutsbekämpfung alter Menschen. Bei ihr werden das gesamte Einkommen und ein das Schonvermögen übersteigendes Vermögen angerechnet;

(3) Mindestrenten, deren Höhe nicht vom übrigen Einkommen oder Vermögen abhängt, aber eine lange Versicherungszeit voraussetzt. Solche Renten können auch durch Aufwertung bestimmter Beitragszeiten in Sozialversicherungssystemen zustande kommen (z. B. Rente nach Mindesteinkommen).

Ausschlaggebend für eine wirksame Vermeidung von Altersarmut ist bei allen drei Formen die Leistungshöhe im Vergleich zur Armutsrisikogrenze.

Im Vergleich zum durchschnittlichen Bruttolohneinkommen von Arbeitnehmern (umgerechnet zu Kaufkraftparitäten), das die OECD ermittelt, lässt sich die relative Höhe dieser Mindestleistungen für eine alte Person darstellen. Abbildung 7 veranschaulicht dies, wobei die maximale Höhe der Mindestleistung als Prozentsatz des durchschnittlichen Bruttolohns und nicht als Absolutbetrag ausgewiesen ist. Diese maximale Höhe kann sich additiv aus zwei gleichzeitig gewährten Mindestleistungen ergeben, oder sie charakterisiert den Betrag der höheren Leistung, die allein gewährt wird. ${ }^{4}$ Die Berechnungen unterstellen aber eine volle Versicherungszeit von 45 Jahren. Fälle kürzerer Versicherungszeit, für die es in vielen Ländern begünstigende Sonderregelungen gibt, sind hier nicht erfasst. Für einige EU-Länder liegen entsprechende Angaben überhaupt nicht vor.

Laut Abbildung 7 sind in den EU-Ländern die Relationen zwischen der höchsten

\section{Abb. 7: Höchste Mindestsicherungsleistung für Alte im Verhältnis zum durchschnittlichen Bruttolohn in Kaufkraftparitäten - in \% -}

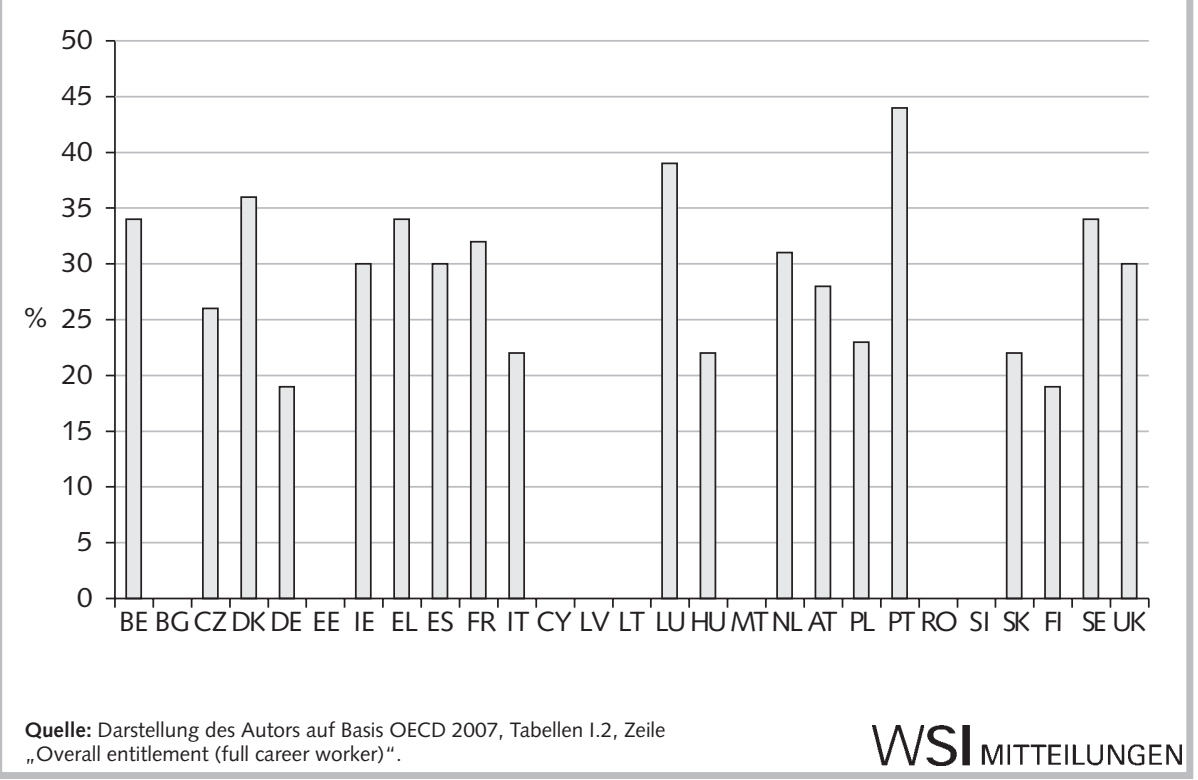

Abb. 8: Mindestleistungen für Alte gemäß OECD und Armutsgrenzen
gemäß EU-SILC in Kaufkraftparitäten im Jahr 2004

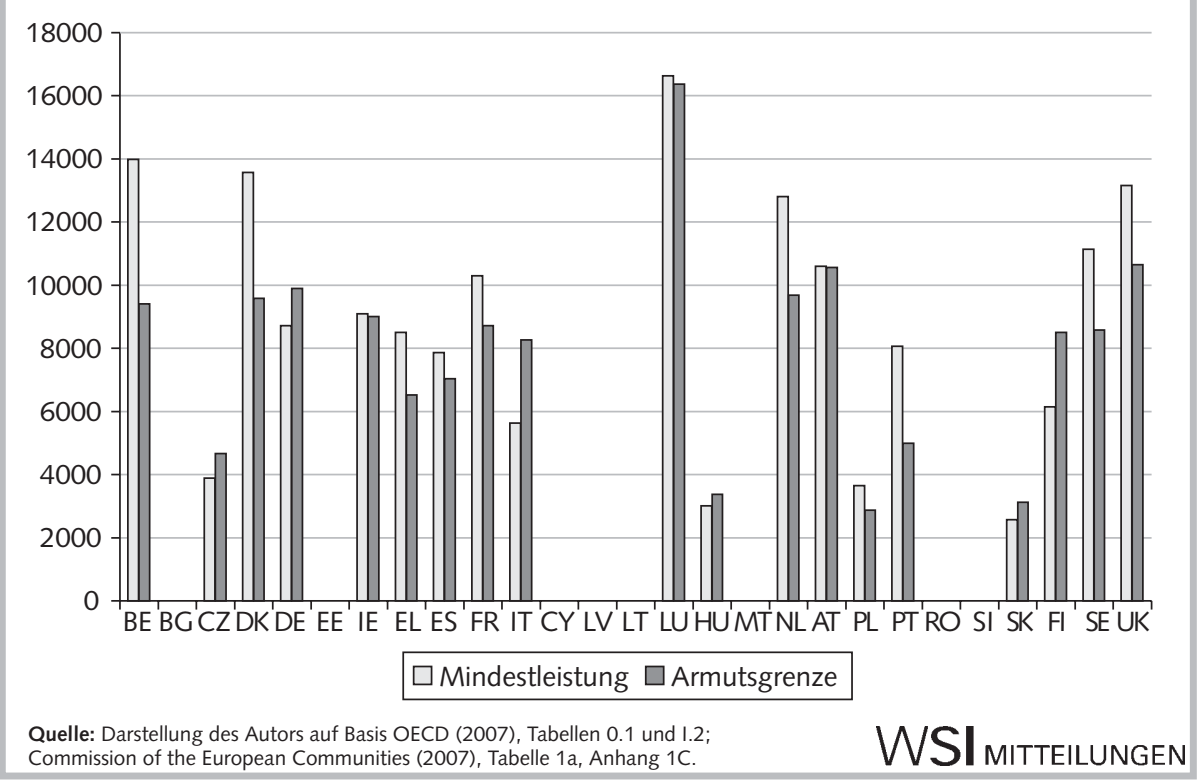

Mindestsicherungsleistung für alleinstehende alte Personen und dem durchschnittlichen Bruttolohn recht unterschiedlich. In den meisten Ländern liegt dieser Wert in einem Bereich um $30 \%$, plus oder minus 5 Prozentpunkte. Aber es gibt einige Ausreißer nach oben: Dänemark, Luxemburg und Portugal. Ebenso findet man einige Länder mit einer Relation von weniger als $25 \%$ : Finnland, Deutschland und die Slowakei. Allerdings darf man diese Angaben nicht ohne Vorbehalte betrachten; denn derartige institutionelle Vergleiche zwi- schen Ländern können viele Feinheiten nicht berücksichtigen.

In Abbildung 8 werden trotz aller Vorbehalte die aus den OECD-Angaben errechneten Höhen der Mindestleistungen für Alte und die von Eurostat auf Basis von EU-SILC abgeleiteten Armutsgrenzen gegenübergestellt. Man erkennt, dass in vielen Ländern die Mindestsicherungsleistung 
für Alte höher ist als die Armutsgrenze. Dies gilt aber nur bei einer „vollen“ Erwerbsbiografie, d.h. 45 Jahre Beitragszahlung, oder bei einer Mindestanzahl an Jahren mit Wohnsitz im Land. Ist diese Bedingung nicht erfüllt, so tritt selbst in den Ländern, in denen die Armutsgrenze niedriger als diese maximale Mindestleistung liegt, Altersarmut auf. In Deutschland aber ist selbst nach 45 Jahren Beitragszahlung die Mindestleistung niedriger als die spezifische deutsche Armutsgrenze.

Die Konstellationen von Einflussfaktoren, die trotz vorhandener Mindestsicherungsregelungen bei vielen Menschen zu Altersarmut führen, müssen noch weiter erforscht werden. Erst dann kann eindeutig beurteilt werden, ob und inwieweit diese Regelungen tatsächlich dazu beitragen, Armut im Alter zu verhindern.

\section{Reformtendenzen der Alterssicherung}

In den letzten Jahren wurden in vielen Mitgliedsländern die Alterssicherungssysteme reformiert, mit dem Ziel, sie nachhaltig und zukunftsfähig zu gestalten. In dem zusammenfassenden Bericht der Europäischen Kommission über die neuen Ergebnisse der Offenen Methode der Koordinierung wird zu den Reformwirkungen festgestellt: Die Nettoersatzraten - das heißt das Verhältnis der Nettorente (abzüglich Sozialbeiträgen) zum vorherigen Nettolohneinkommen - werden in Zukunft in zwölf Mitgliedsländern sinken; in acht wird dieses Verhältnis voraussichtlich gleich bleiben und in sechs sogar leicht ansteigen. Angaben zu den restlichen Ländern fehlen. Wegen der Anpassungsregeln werden die Bestandsrenten aber im Vergleich zum Zuwachs der Nettoeinkommen nur unterproportional zunehmen, sodass die Ersatzraten mit steigendem Lebensalter der Rentner zusätzlich absinken, d. h. die Rentner werden in vielen Ländern mit ihrem Lebensstandard immer weiter hinter den
Erwerbstätigen zurückbleiben (Commission of the European Communities 2007, Tabelle A 3).

Hinzu kommen weitere Probleme:

- Häufigere Unterbrechungen von Erwerbskarrieren;

- ein zunehmender Niedriglohnsektor;

- die Einführung strengerer Bindungen der Rentenleistungen an die vorher gezahlten Beiträge, d h. eine Verstärkung des Äquivalenzprinzips in der Altersvorsorge; - ein stärkerer Verweis auf eine Absicherung durch private Vorsorge, bei der generell die auf dem Solidarprinzip beruhenden umverteilenden Elemente in der staatlichen Pflichtrentenversicherung ausgeschlossen sind;

- weitere Zuwanderung von Personen, die keine dem Zuwanderungsalter entsprechenden ausreichenden Alterssicherungsansprüche mitbringen; immerhin gehen die offiziellen Bevölkerungsvorausschätzungen von einer jährlichen Zuwanderung von 200.000 Personen aus.

Alle diese Faktoren werden dazu führen, dass es den Pflichtalterssicherungssystemen künftig immer weniger gelingen wird, ohne eine Verbesserung der Mindestsicherungsregelungen Altersarmut zu vermeiden oder wenigstens zu begrenzen.

Bereits im Jahr 1992 hat der Rat der Europäischen Gemeinschaften eine Empfehlung über gemeinsame Kriterien für ausreichende Zuwendungen und Leistungen im Rahmen der Sozialsysteme beschlossen. Wenn die Mitgliedsländer auch noch weit von der vollen Realisierung dieser Empfehlung entfernt sind, so haben sie doch in den letzten Jahren im Bereich der Mindestsicherungsregelungen wichtige Reformen durchgeführt (Commission of the European Communities 2007, S. 122ff.). In Belgien, Frankreich und Finnland wurden die Mindestleistungen stärker erhöht als die Renten des staatlichen Systems. In Irland, Spanien und Portugal wurden die Mindestleistungen sogar stärker erhöht als die Löhne gestiegen sind. Andere Mitgliedsstaaten haben neue Mindestsicherungsre- gelungen eingeführt oder alte tiefgreifend verändert. Hierzu zählen das Vereinigte Königreich, Portugal, Litauen und die Slowakei. In weiteren Ländern wurden Ergänzungsleistungen zu bestehenden Sozialleistungen für Alte eingeführt; dies geschah in Dänemark und in Ungarn. In Deutschland wurde die Bedarfsorientierte Grundsicherung im Alter und bei Erwerbsminderung teilweise aus der Sozialhilfe herausgelöst und der Zugang zu diesem Sicherungssystem dadurch erleichtert, dass die Kinder alter Menschen von der bisherigen Kostenerstattungspflicht weitgehend entbunden wurden. Diese Reformen haben die Altersarmut reduziert oder zumindest die Armutslücke verringert.

Aber diese Reformen sind nicht hinreichend, um die skizzierten Auswirkungen der künftigen Verschlechterung der Alterssicherungsleistungen einzudämmen. Damit werden Mindestsicherungsregelungen zunehmend an Bedeutung gewinnen. Sie können allerdings Altersarmut in allen Mitgliedsländern der EU nur vermeiden oder zumindest stark reduzieren, wenn

(1) die Voraussetzungen für die Inanspruchnahme der Mindestsicherungsleistungen im Alter derart gestaltet werden, dass sie jeder alte Mensch mit zu niedrigem Einkommen auf der Basis eines Rechtsanspruchs erhält;

(2) eine Harmonisierung der Mindestsicherungsregelungen in den EU-Mitgliedsländern auf dem gleichen relativen Niveau in Höhe der EU-Armutsrisikogrenze stattfindet;

(3) in jedem Mitgliedsland ein parallel mit den durchschnittlichen Nettoeinkommen ansteigendes Niveau der Mindestsicherungsleistungen gewährleistet wird.

Es bleibt eine offene Frage, ob es den Mitgliedsländern gelingt, den zu erwartenden Zuwachs an Altersarmut zu begrenzen. Die Instrumente sind vorhanden. 
Atkinson, A. B. (1998): Poverty in Europe, Oxford

Atkinson, A. B./Cantillon, B./Marlier, E./Nolan, B. (2002): Social Inclusion: The EU and Social Inclusion, Oxford

Bundesministerium für Arbeit und Sozialordnung (BMAS) (2001):

Lebenslagen in Deutschland. Der erste Armuts- und Reichtumsbericht der Bundesregierung, Bonn

Bundesregierung (2005): Lebenslagen in Deutschland. Der zweite Armuts- und Reichtumsbericht der Bundesregierung, Bonn

Commission of the European Communities (2007): Joint Report on Social Protection and Social Inclusion, Commission Staff Working Document, Brussels (SEC 2007) 329 vom 6. 3.

Eichenhofer, E. (2007): Geschichte des Sozialstaats in Europa. Von der "sozialen Frage" bis zur Globalisierung, München

Hauser, R. (1993): Mindestsicherung im Alter - Ausgewählte ökonomische Aspekte unter Berücksichtigung von Wanderungen, in: Schmähl, W. (Hrsg.): Mindestsicherung im Alter - Erfahrungen, Herausforderungen, Strategien, Frankfurt a. M./New York, S. 75-99

Hauser, R. (1995): Stand und Entwicklungstendenzen der Annäherung der sozialen Sicherung in der europäischen Union: Das Beispiel Alterssicherung, in: Schmähl, W./Rische, H. (Hrsg.): Internationalisierung von Wirtschaft und Politik - Handlungsspielräume der nationalen Sozialpolitik, Baden-Baden, S. 139-182

Hauser, R. (1996): Zur Messung individueller Wohlfahrt und ihrer Verteilung, in: Statistisches Bundesamt (Hrsg.): Wohlfahrtsmessung - Aufgabe der Statistik im gesellschaftlichen Wandel, Wiesbaden, S. 13-38

Hauser, R. (1996): Are Pension Systems Converging in the European Union?, in: Ritter, U. P. (Hrsg.): Problems of Structural Change in the 21st Century, National and Comparative Research from Argentina, Brasil and Germany, Frankfurt a. M., S. 320-347
Hauser, R. (1997): Soziale Sicherung in westeuropäischen Staaten, in: Hradil, S./Immerfall, S. (Hrsg.): Die westeuropäischen Gesellschaften im Vergleich, Opladen, S. 521-545

Hauser, R. (1999): Mindestregelungen für die Alterssicherung und Armut unter den Älteren in den EU-Ländern, in: Flora, P./Noll, H.-H. (Hrsg.): Sozialberichterstattung und Sozialstaatsbeobachtung - Individuelle Wohlfahrt und wohlfahrtsstaatliche Institutionen im Spiegel empirischer Analysen, Frankfurt/New York, S. 141-167

Hauser, R. (2002): Soziale Indikatoren als Element der offenen Methode der Koordinierung zur Bekämpfung von Armut und sozialer Ausgrenzung in der EU, in: Zeitschrift für Sozialreform 3, S. $251 \mathrm{ff}$.

Hauser, R./Strengmann-Kuhn, W. (2002): Armut der älteren Bevölkerung in den Ländern der Europäischen Union, DRV-Schriften Band 54, Berlin Huster, E.-U. (1996): Armut in Europa, Opladen

Marlier, E./Atkinson, A. B./Cantillon, B./Nolan, B. (2007): The EU and Social Inclusion. Facing the Challenges, Bristol OECD (2007): Pensions at a Glance. Public Policies across OECD Countries, Paris

Europäische Kommission, GD Beschäftigung und Soziales (Hrsg.) (2004): MISSOC 2004, Gegenseitiges Informationssystem zur sozialen Sicherheit, Luxemburg

Verband Deutscher Rentenversicherungsträger (VDR) (Hrsg.) (2005): OECD: Renten auf einen Blick: Staatliche Politik im OECD-Ländervergleich, DRV-Schriften, Bd. 61, Berlin 